\title{
Adomian Decompostion Method for Solving Ratio-Dependent Prey- Predator System with Harvesting on Predator
}

\author{
V. Madhusudanan ${ }^{1, a^{*}}$, P.N. Duraiswamy ${ }^{2, b}$, S. Vijaya ${ }^{3, c}$ \\ ${ }^{1 *}$ Department of Mathematics, S.A Engineering College, Chennai-600077, Tamilnadu,India \\ ${ }^{2}$ Kothandaraman High School, Utthu Kottai, Tamilnadu, India \\ ${ }^{3}$ Department of Mathematics, Annamalai University, Tamilnadu, Annamalainagar, Chidambaram- \\ 608002 \\ amvms.maths@gmail.com, bdrpnduraiswamy@gmail.com, chavenksho@gmail.com
}

Keywords: Adomian Decomposition Method, Ratio-dependent Prey-Predator model, Predator harvesting

\begin{abstract}
In this article, Adomian Decomposition Method is used to find out the approximate solution of ratio-dependent prey-predator model with predator harvesting. The significance of ADM over other numerical discretization techniques is that it has the eminence to solve problems directly with no un-physical restrictive assumptions such as linearization, per-turbation, massive computation and any other transformation. ADM solves the problem and arrives at the approximate solution in the form of series with solution components that are easily computable. It requires very less work in comparison with other traditional methods. The graphical representations of prey and predator population contrasted with time are drawn to examine the performance and reliability of this technique.
\end{abstract}

\section{Introduction}

Many biological systems are based on the system of non-linear ordinary differential equations. The qualitative and quantitative analysis of these systems can be done by mathematical modeling. In order to mathematically examine ecology, it is significant to design models that involve the interaction between species. There are many discrete and continuous prey-predator models in mathematical ecology. These models are investigated for global stability, periodicity, boundedness and other features [2,3]. One of the most well-known models is the prey-predator model developed by Lotka-Volterra [7].

Adomian Decomposition Method is a powerful approach to solve non-linear differential equations by substitution of the non-linear part of the equation with Adomian Polynomials. A step by step procedure is used for finding the solution. The number of partial solutions used decides the accuracy of the system. It is noteworthy that the solution of this method has a fast convergence to the exact solution. The numerical solution of prey - predator model has been treated in several papers in order to numerically investigate reliability and efficiency. For instance, in [6 9, 10], Adomian has been tested numerically using prey-predator model. The analysis of ratio-dependent predator-prey model by Homotopy method is done by $[1,12]$.

The objective of this paper is to numerically examine the reliability of ADM applied to ratio-dependent prey-predator models governed by a two dimensional system of non-linear ordinary differential equation. In section (2), the mathematical model of the system is analyzed. Section (3) presents the basics of Adomian Decomposition Method. In section (4), the computational method for the considered system is discussed. Section (5) consists of the numerical simulation and the it's result. Section (6) concludes the work. 


\section{Mathematical Model}

The general case of predator- prey system is as follows:

$$
\begin{aligned}
& \frac{d x}{d t}=x h(x)-y p(x, y) \\
& \frac{d y}{d t}=\beta y p(x, y)-\gamma y
\end{aligned}
$$

Where $x(t)$ and $y(t)$ represents the size of the prey and predator population respectively at time $\mathrm{t}, \mathrm{h}(\mathrm{x})$ is the per-capita growth rate in the absence of the predator, $p(x, y)$ is the functional response $\beta p(x, y)$ is the per-capita production of predator due to predation which is often called the numerical response, $\gamma$ is the natural mortality of predator. The functional response plays a major role in system (1). The knowledge of this function determines the dynamics of the whole system and the transfer of bio-mass in the predation because it is proportional to numerical response. Now by letting $h(x)=(1-x), p(x, y)=\frac{x}{x+y} \quad$ we obtain the special case of predator-prey system called predator-prey system with constant effort harvesting on predator which formulated as follows:

$$
\begin{aligned}
& \frac{d x}{d t}=x(1-x)-\frac{\alpha x y}{x+y} \\
& \frac{d y}{d t}=\frac{\beta x y}{x+y}-\gamma y-h y
\end{aligned}
$$

Here $\alpha>0$ and $h>0$ represents capturing rate and harvesting activity respectively. Equations (2) and (3) are to be solved according to biological meaningful initial conditions $x(t) \geq 0, y(t) \geq 0 \mathrm{~A}$ qualitative examination of the system described by equations (2) and (3) reveals that the long term behavior falls into two categories: predator extinction and co-existence [1]. When the predator population becomes extinct, the solution asymptotically approaches equilibrium $P_{1}$ of the form

$$
P_{1}=(1,0)
$$

The eigen values of the Jacobian matrix evaluated at equilibrium $P_{1}$ shows that the predator extinction equilibrium is a locally asymptotically stable node provided if the death rate combined with harvesting of the predator exceeds the conversion rate(i.e.) $\gamma+h>\beta$.

Another long term possibility is the prey-predator co-existence equilibrium $P_{2}$ of the form

$$
P_{2}=\left(\frac{\beta(1-\alpha)+\alpha(\gamma+h)}{\beta}, \frac{\beta-\gamma-h}{\gamma+h}\left(\frac{\beta(1-\alpha)+\alpha(\gamma+h)}{\beta}\right)\right)
$$

The eigen value $\lambda$ of the Jacobian matrix evaluated at $P_{2}$ satisfy

$$
\lambda^{2}-(\alpha-\gamma-h-1)+\left[\frac{(\gamma+h)^{2}(\beta-\alpha)}{\beta^{2}}\right] \lambda+\left[\frac{(\gamma+h)(\beta-\gamma-h)(\beta-\beta \alpha+\alpha \gamma+\gamma h)}{\beta^{2}}\right]
$$

Hence $P_{2}$ is locally asymptotically stable provided

$$
\beta>\gamma+h \text { and } \alpha<\beta\left(\frac{(\gamma+h+1) \beta-(\gamma+h)^{2}}{\beta^{2}-(\gamma+h)^{2}}\right)
$$


From equation (5), we observe that wherever co-existence equilibrium $P_{2}$ occurs, the predator extinction equilibrium $P_{1}$ becomes an unstable saddle point. This is because the predator conversion rate exceeds the sum of death and harvest rate.

\section{Basics Adomian Decomposition Method}

Consider the standard operator

$$
L u+R u+N u=g
$$

Where $\mathrm{u}$ is the unknown function, $\mathrm{L}$ is the highest order derivative which is assumed to be easily invertible, $\mathrm{R}$ is a linear differential operator of order less than the order of $\mathrm{L}, \mathrm{Nu}$ represents the nonlinear terms and $\mathrm{g}$ is the source term. By applying the inverse operator $L^{-1}$ to both sides of equation () we obtain

$$
u=v-L^{-1}(R u)-L^{-1}(N u)
$$

Where the function $\mathrm{v}$ represents the terms arising from integrating the source term $\mathrm{g}$ and from using the auxiliary conditions. The standard Adomian method defines the solution $u$ by the series

$$
u=\sum_{n=0}^{\infty} u_{n}
$$

And the non-linear term by the series

$$
N u=\sum_{n=0}^{\infty} A_{n}
$$

Where $A_{n}$ are the Adomian Polynomials determined formally from the relation [ ]

$$
A_{n}=\frac{1}{n !}\left[\frac{d^{n}}{d \lambda^{n}}\left[N\left(\sum_{i=0}^{\infty} \lambda^{i} u_{i}\right)\right]\right]_{\lambda=0}, n=0,1, \ldots,
$$

The solution components $u_{0}, u_{1}, u_{2}, \ldots$, are then determined recursively by using the relation

$$
\left\{\begin{array}{l}
u_{0}=v \\
u_{k+1}=-L^{-1} R u_{k}-L^{-1} A_{k}, k \geq 0
\end{array}\right\}
$$

Where $u_{0}$ is referred to as zeroth component.

\section{Computational method}

In order to explicitly construct approximate non-perturbative solution of the system described by the (2) and (3), Adomian Decomposition which is well addressed in $[4,5,8,13]$ is employed. The advantage of this method is that it provides a direct scheme for solving the problem. This decomposition method is also an approximation but does not change the original problem. Another advantage of decomposition method is that it provides analytical approximation to a wide class of non-linear equations without any linearization as in perturbation, massive computation and any other transformation.

Now

$$
\frac{d x}{d t}=x(t)-x^{2}(t)-\frac{a x(t) y(t)}{x(t)+y(t)}
$$




$$
\frac{d y}{d t}=\frac{\beta x(t) y(t)}{x(t)+y(t)}-(\gamma+h) y(t)
$$

Considering $\mathrm{f}$ and $\mathrm{g}$ to be a nonlinear functions of $x$ and $(x, y)$ respectively. The equation (8-9) can now written in the form

$$
\begin{aligned}
& \frac{d x}{d t}=x(t)-f(x)-\alpha g(x, y) \\
& \frac{d y}{d t}=\beta g(x, y)-(\gamma+h) y(t)
\end{aligned}
$$

With initial values $x(0)=x_{0}, y(0)=y_{0}$. To find the solution of $x$ and $y$ that satisfying equation (10) and (11), the decomposition method is used. It consists of approximating solution of (10) and (11) as an infinite series in the following form

$$
x=\sum_{n=0}^{\infty} x_{n}, \quad y=\sum_{n=0}^{\infty} y_{n}
$$

Decomposing $f$ and $g$ as

$$
\begin{aligned}
f(x) & =\sum_{n=0}^{\infty} A_{n}\left(x_{0} \ldots \ldots \ldots x_{n}\right) \\
g(x, y) & =\sum_{n=0}^{\infty} B_{n}\left(x_{0} \ldots \ldots \ldots x_{n}, y_{0} \ldots \ldots \ldots \ldots \ldots y_{n}\right)
\end{aligned}
$$

Where $A_{n}, B_{n}$ are called Adomian polynomials that can be determined formally from the relation

$$
\begin{aligned}
& A_{n}=\frac{1}{n !}\left[\frac{d^{n}}{d \lambda^{n}}\left[\left(\sum_{i=0}^{\infty} \lambda^{i} x_{i}\right)\right]\right]_{\lambda=0}, n=0,1, \ldots, \\
& B_{n}=\frac{1}{n !}\left[\frac{d^{n}}{d \lambda^{n}}\left[\left(\frac{\sum_{i=0}^{\infty} \lambda^{i} x_{i} \sum_{i=0}^{\infty} \lambda^{i} y_{i}}{\sum_{i=0}^{\infty} \lambda^{i} x_{i}+\sum_{i=0}^{\infty} \lambda^{i} y_{i}}\right)\right]\right]_{\lambda=0}, n=0,1, \ldots,
\end{aligned}
$$

Applying decomposition method, the system (10) and (11) can be written as

$$
\begin{aligned}
& L x=x(t)-f(x)-\alpha g(x, y) \\
& L y=\beta g(x, y)-(\gamma+h) y(t)
\end{aligned}
$$

Where $L=\frac{d}{d t}$, the linear differential operator. Applying the integration inverse operator $L^{-1}=\int_{0}^{t}() d$.$t to both sides of equation (17) and (18) we get$

$$
\begin{aligned}
& x(t)=x(0)+L^{-1} x(t)-L^{-1} f\left(x(t)-\alpha L^{-1} g(x(t), y(t))\right. \\
& y(t)=y(0)+\beta L^{-1} g(x(t), y(t))-(\gamma+h) L^{-1} y(t)
\end{aligned}
$$

Using equation (12), (13) and (14) 


$$
\begin{aligned}
& \sum_{n=0}^{\infty} x_{n}=x_{0}+L^{-1} \sum_{n=0}^{\infty} x_{n}-L^{-1} \sum_{n=0}^{\infty} A_{n}-\alpha L^{-1} \sum_{n=0}^{\infty} B_{n} \\
& \sum_{n=0}^{\infty} y_{n}=y_{0}+\beta L^{-1} \sum_{n=0}^{\infty} B_{n}-(\gamma+h) L^{-1} \sum_{n=0}^{\infty} y_{n}
\end{aligned}
$$

Now, the iterates are determined in the following recursive way

$$
\begin{aligned}
& x(0)=x_{0} \\
& x_{n+1}=L^{-1} x_{n}-L^{-1} A_{n}-\alpha L^{-1} B_{n}, n=0,1,2 \\
& y(0)=y_{0} \\
& y_{n+1}=\beta L^{-1} B_{n}-(\gamma+h) L^{-1} y_{n}, n=0,1,2
\end{aligned}
$$

We define a solution of initial value problem as

$$
(x, y)=\left(\lim _{n \rightarrow \infty} \sum_{n=0}^{n} x_{n}, \lim _{n \rightarrow \infty} \sum_{n=0}^{n} y_{n}\right)
$$

Computing Adomian polynomials by using relation the algorithm presented in[4] yields to

$$
\begin{aligned}
& A_{0}\left(x_{0}\right)=x_{0}^{2} \\
& A_{1}\left(x_{0}, x_{1}\right)=2 x_{0} x_{1} \\
& A_{2}\left(x_{0}, x_{1}, x_{2}\right)=2 x_{0} x_{2}+x_{1}^{2} \\
& A_{3}\left(x_{0}, x_{1}, x_{2}, x_{3}\right)=2 x_{0} x_{3}+2 x_{2} x_{1}
\end{aligned}
$$

and

$$
\begin{aligned}
& B_{0}\left(x_{0}, y_{0}\right)=\frac{x_{0} y_{0}}{x_{0}+y_{0}} \\
& B_{1}\left(x_{0}, x_{1}, y_{0}, y_{1}\right)=\frac{x_{1} y_{0}{ }^{2}+x_{0}{ }^{2} y_{1}}{\left(x_{0}+y_{0}\right)^{2}} \\
& B_{2}\left(x_{0}, x_{1}, x_{2}, y_{0}, y_{1}, y_{2}\right)=\frac{x_{2} y_{0}{ }^{3}+x_{0} x_{2} y_{0}{ }^{2}+2 x_{1} x_{0} y_{0} y_{1}-x_{1}{ }^{2} y_{0}{ }^{2}+x_{0} y_{2} y_{0}+x_{0}{ }^{3} y_{2}-x_{0}{ }^{2} y_{1}{ }^{2}}{\left(x_{0}+y_{0}\right)^{3}}
\end{aligned}
$$

Four terms approximations to the solutions are considered

$$
\left\{\begin{array}{l}
x(t)=x_{0}+x_{1}+x_{2}+x_{3} \\
y(t)=y_{0}+y_{1}+y_{2}+y_{3}
\end{array}\right\}
$$

\section{Numerical simulation and results}

In this section we present some graphical results based on the decomposition method together with numerical validation of the qualitative results obtained in equation (2) and (3). According to the values introduced in Table 1, the following decomposition series with partial sums are derived.

Case1:

$$
\begin{aligned}
& x(t) \approx 0.5+0.175 t-0.027046 t^{2}+0.0205461313 t^{3} \\
& y(t) \approx 0.3-0.3525 t+0.22791414 t^{2}-0.0993106909 t^{3}
\end{aligned}
$$


Case 2:

$$
\begin{aligned}
& x(t) \approx 0.4+0.17152857 t+0.09798022 t^{2}-0.001987925 t^{3} \\
& y(t) \approx 0.3-0.1328571 t+0.01300744 t^{2}-0.00248976 t^{3}
\end{aligned}
$$

Case 3:

$$
\begin{aligned}
& x(t) \approx 0.3+0.15857 t+0.02536 t^{2}-0.00514075 t^{3} \\
& y(t) \approx 0.4-0.051432 t+0.01618104 t^{2}-0.0014306125 t^{3}
\end{aligned}
$$

Case4:

$$
\begin{aligned}
& x(t) \approx 0.3+0.17 t+0.028 t^{2}-0.004138683 t^{3} \\
& y(t) \approx 0.6-0.14 t+0.04 t^{2}-0.009736627 t^{3}
\end{aligned}
$$

\begin{tabular}{|l|l|l|l|l|l|l|l|}
\hline Case & $x_{0}$ & $y_{0}$ & $\alpha$ & $\beta$ & $\gamma$ & $h$ & Comments \\
\hline 1 & 0.5 & 0.3 & 0.4 & 0.2 & 0.5 & 0.8 & Stable- predator extinction \\
\hline 2 & 0.4 & 0.3 & 0.4 & 0.1 & 0.2 & 0.3 & Stable predator extinction \\
\hline 3 & 0.3 & 0.4 & 0.3 & 0.4 & 0.2 & 0.1 & Stable-coexistence \\
\hline 4 & 0.3 & 0.6 & 0.2 & 0.5 & 0.2 & 0.2 & Stable- co-existence \\
\hline
\end{tabular}

Fig 1-4 shows the relation between prey and predator population contrasted with time.

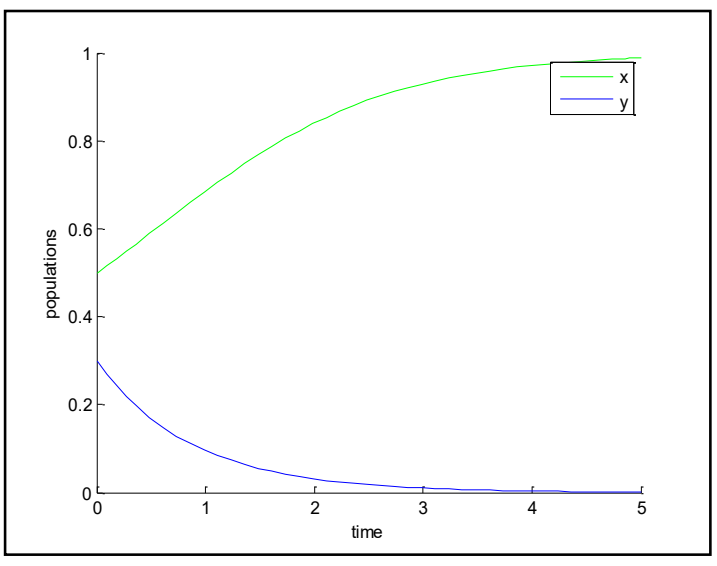

Fig 1. Case 1: For $\mathrm{h}=0.8$

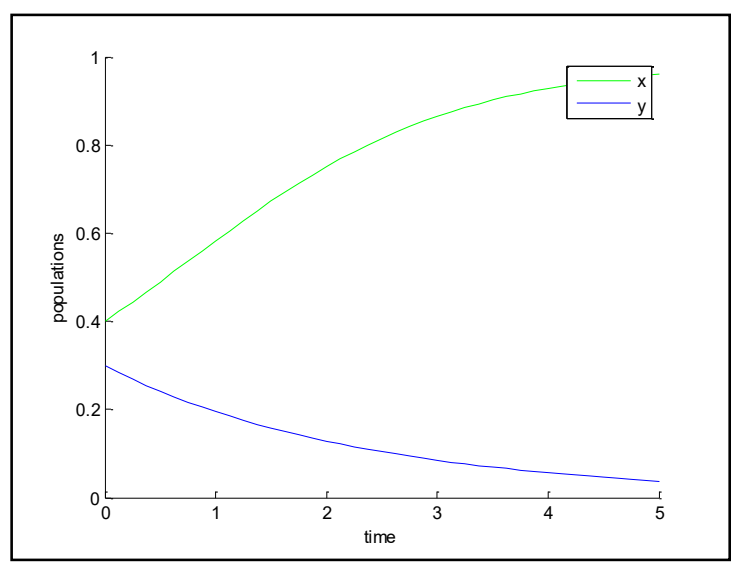

Fig 2. Case 2: For $\mathrm{h}=0.3$

A noteworthy observation from Fig 1 and 2 is that predator population gradually decreases and becomes extinct despite the availability of increasing prey population. It can be observed that if the sum of death rate and harvesting rate of the predator exceeds its conversion rate, then the predator will go extinct.

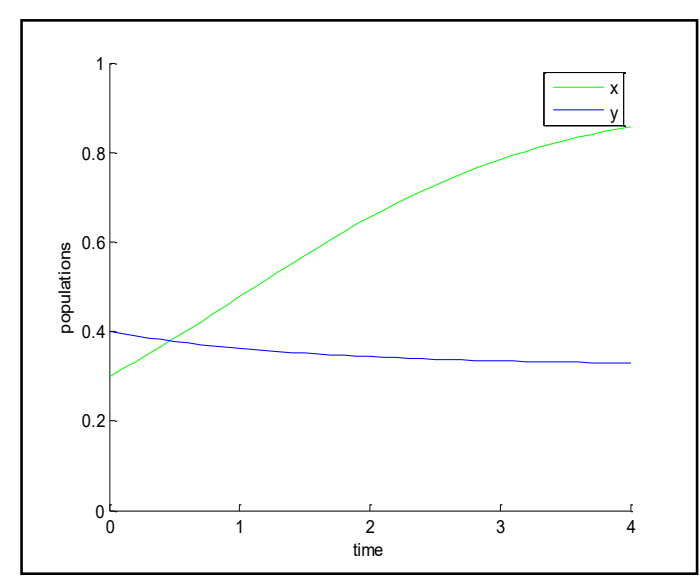

Fig 3. Case 3: For $\mathrm{h}=0.1$

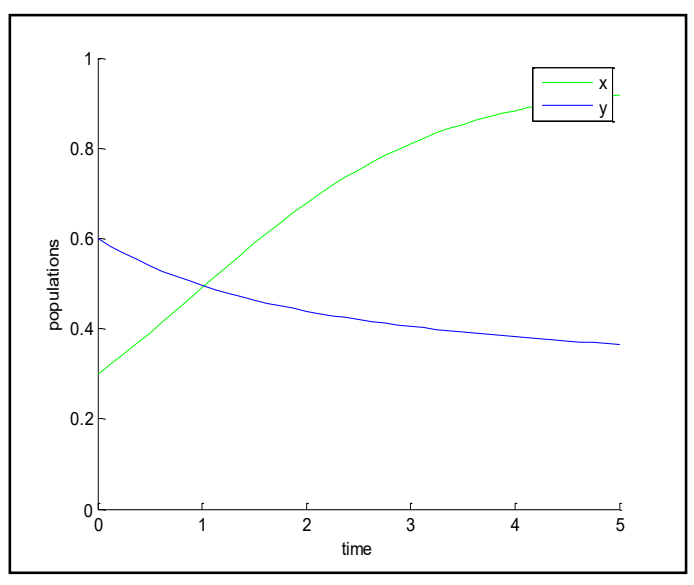

Fig 4. Case 4: For $\mathrm{h}=0.2$ 
Fig 3 and 4 illustrate the possibility of long time co-existence of predator and prey. The predator conversion rate must exceed the sum of death rate and harvest rate for co-existence to occur among the other necessary conditions

\section{Conclusion}

In this paper, the approximate solution of ratio-dependent prey-predator system is simulated by Adomian Decomposition Method in a direct way without using any restrictive assumption. Therefore, the computation of work and consumption of time is reduced. The performance and reliability of this method is analyzed from the graphical representation of prey and predator population plotted against time.

\section{Reference}

[1] Abdoul R. Ghotbi,A. Barari and D. D. Ganji," Solving Ratio-Dependent Predator-Prey System with Constant Effort Harvesting Using Homotopy Perturbation Method", Hindawi Publishing Corporation ,Volume 2008, Article ID 945420, 8 pages,doi:10.1155/2008/945420.

[2] D.K. Arrowsmith, C.M..Place,Ordinary Differential Equations, Chapman and Hall, 1982.

[3] Francisco J. Solis. Self -limitation in a discrete predator-prey model, Mathematical and Computer Modelling, 48, 2008, (1-2) :pp.191-196.

[4] G.Adomian, A global method for solution of complex systems ,Math. Model 5 (1984) 521-568.

[5] G.Adomian, Solving Frontier Problems of Physics :The Decomposition Method, Kluwer Academic Publishers, Dordecht, 1994.

[6] J.Biazar and R.Montazeri, A Computational method for solution of the prey and predator problem, Applied Mathematics and Computation, 163, 2005, (2): pp.841-847.

[7] J.D.Murray, Mathematical Biology: I. An Introduction,Springer,Berlin,2002.

[8] K.Abboui,Y.Cherruault,New ideas for proving convergence of decomposition methods, Comput.Appl.Math .29 (7) (1995) 103-105.

[9] Lazhar Bougoffa, Solvability of the predator and prey system with variable coefficients and comparison of the results with modified decomposition, Applied Mathematics and Computation, 182, 2006, (1):pp.383-387.

[10]M.S.H. Chowdhury, I.Hashim and S.Mawa, Solution of prey predator problem by numeric analytic technique, Communications in Nonlinear Science and Numerical Simulation ,14, 2009, (4): pp. 10081012.

[11] O. D. Makinde, "Solving ratio-dependent predator-prey system with constant effort harvesting using Adomian decomposition method," Applied Mathematics and Computation, vol. 186, no. 1, pp. 17-22,2007

[12] Safar Irandoust, Ahmad Golbabai, Hosein Kheiri and Davood Ahmadian," Homotopy analysis method for solving ratio-dependent predator-prey system with constant effort harvesting by using two parameters h1 and h2", Acta Universitatis Apulensis ,2011, 327-340.

[13] V.Daftardar-Gejji ,H.Jafari ,Adomian decomposition a tool for solving a system of fractional differential equation ,J.Math .Anal.Appl.301 (2) (2005) 508-518. 